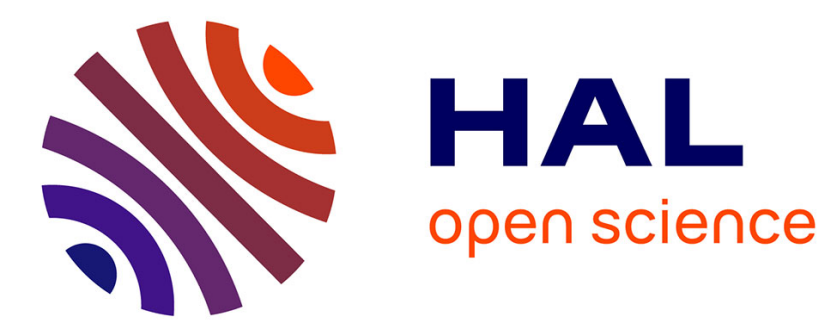

\title{
Optical flow estimation from multichannel spherical image decomposition
}

\author{
Amina Radgui, Cédric Demonceaux, El Mustapha Mouaddib, Mohamed \\ Rziza, Driss Aboutajdine
}

\section{- To cite this version:}

Amina Radgui, Cédric Demonceaux, El Mustapha Mouaddib, Mohamed Rziza, Driss Aboutajdine. Optical flow estimation from multichannel spherical image decomposition. Computer Vision and Image Understanding, 2011, 115 (9), pp.1263-1272. hal-00637423

\section{HAL Id: hal-00637423 https://hal.science/hal-00637423}

Submitted on 3 May 2018

HAL is a multi-disciplinary open access archive for the deposit and dissemination of scientific research documents, whether they are published or not. The documents may come from teaching and research institutions in France or abroad, or from public or private research centers.
L'archive ouverte pluridisciplinaire HAL, est destinée au dépôt et à la diffusion de documents scientifiques de niveau recherche, publiés ou non, émanant des établissements d'enseignement et de recherche français ou étrangers, des laboratoires publics ou privés. 


\title{
Optical flow estimation from multichannel spherical image decomposition
}

\author{
A. Radgui ${ }^{1 \text { 出 }}$, C. Demonceaux ${ }^{2}$, E. Mouaddib $^{2}$, M. Rziza $^{1}$, D. Aboutajdine ${ }^{1}$ \\ ${ }^{1}$ LRIT associated unit with CNRST, Mohammed V-Agdal University, B.P 1014, Rabat, \\ Morroco. \\ 2 M.I.S. University of Picardie Jules Verne, 33, rue Saint Leu, 80039 Amiens, France.
}

\begin{abstract}
The problem of optical flow estimation is largely discussed in computer vision domain for perspective images. It was also proven that, in terms of optical flow analysis from these images, we have difficulty distinguishing between some motion fields obtained with smooth camera motion. The omnidirectional cameras developed recently provided images with large filed of view. These images will contain global information about motion and allows to remove the ambiguity present in the case of planar projection in terms of optical flow analysis. Nevertheless, these images contain significant radial distortions that is necessary to take into account when treating these images to estimate the motion. In this paper, we shall describe new way to compute efficient optical flow for several camera motions given synthetic and real omnidirectional images. Our formulation of optical flow estimation problem will be given in the spherical domain. The omnidirectional images will be mapped on the sphere and used in multichannel image decomposition
\end{abstract}

\footnotetext{
${ }^{\star}$ Corresponding author.

This work is supported by the project MA/07/174, PAI: VOLUBILIS.
} 
process to constraint spherical optical flow equation. This decomposition is based on spherical wavelets. The optical flow fields obtained using our proposed approach are illustrated and compared to that obtained with multichannel image decomposition method developed for perspective images and other published methods dedicated to omnidirectional images.

Keywords: Omnidirectional images, optical flow, spherical wavelets

\section{INTRODUCTION}

Optical flow estimation is one of the basic problems in computer vision. It is the projection of the $3 \mathrm{D}$ velocity on the image and can be extracted from the image brightness variation. It was used by robotics researchers in many tasks such as : object detection and tracking, motion detection, ego-motion estimation, robot navigation or visual odometry (see for example [1] [2] [3] [4] [5] [6] and references therein).

Given an image sequence $I(x, y, t)$, the optical flow estimation consists in measuring the velocity $\left(v_{x}, v_{y}\right)$ of pixel $(\mathrm{x}, \mathrm{y})$ between $t$ and $t+1$. Assuming the brightness constant across successive frames, this velocity can be computed by the well-known brightness constancy constraint equation (BCCE) eq.(1):

$$
\frac{\partial I}{\partial x} \cdot v_{x}+\frac{\partial I}{\partial y} \cdot v_{y}+\frac{\partial I}{\partial t}=0
$$

No unique solution of eq.(1) is possible, since on each location and each time, we have to solve a single equation to determine two scalar unknowns $\left(v_{x}, v_{y}\right)$. This was called the "aperture problem". Equation (1) only provides the normal velocity component. To overcome this problem more than one equation 
is needed to obtain the two velocity components at each position $(x, y)$. For traditional cameras, a number of different approaches to recovering optical flow have been proposed by adding other hypotheses of the flow. Horn and Schunck [7] are the first to introduce a smoothness constraint in order to solve the aperture problem. Later a number of approaches are proposed in different frameworks. These methods can be grouped into correlation [8], energy [9] [10], phase [11] [12] [13], differential [7] [14] [15] and multichannel decomposition approaches [16][17][18] [19]. It should be noted that the differential method proposed by Lucas and Kanade [14], even if it is an old method, is the most commonly used in robotics applications since it is a simple method and often gives acceptable results. A review of a number of the most popular optical flow techniques can be found in [20] where the different approaches were compared. In this review, Fleet et al. [20] found that a phase-based approach [11] performed the best numerically than energy [9] [10], region-matching [8] and differential approaches [7] [14] [15]. Later, it was proven that the multichannel decomposition of the optical flow equation gives robust estimation [17] [18] [19]. The multichannel image decomposition methods give a good compromise between robustness and time computation. Recently, a new evaluation methodology and benchmarks for optical flow estimation methods in perspective images is proposed in [21]. It replace the quantitative evaluation of optical flow given by Barron et al. [20]. It gives set of databases and comparison of several well-known methods.

Referring to all these works, the problem of optical flow estimation was largely discussed in computer vision domain for perspective images. It was also proved that estimation methods from these images have difficulty distin- 
guishing between small pure translations and small pure rotations. Recently, the developed omnidirectional cameras with large field of view have been able to overcome the limited field of view introduced by planar projections [6]. The omnidirectional images with hemispherical field of view contain global information about motion with the presence of the focus of expansion (FOE) and/or the focus of contraction (FOC) in the images. Consequently, the optical flow filed analysis from omnidirectional images is more efficient also with smooth camera motion.

The catadioptric images are obtained using a combination of lenses and mirrors. These images are distorted due to the non-linear projection of the scene points in the image. Thus, the traditional treatments developed for perspective images are not appropriate for the deformed catadioptric images. Different works tried to adapt the existing methods by proposing an adaptation of the concepts definition of neighborhood, gradient function, Gaussian function, and so forth... We may classify the proposed works into two different approaches:

- Optical flow computation directly on the catadioptric image: A method to estimate optical flow on the catadioptric images was proposed on [22] using wavelet approach based on a brightness change constraint equation. This method does not take into account the radial distorsion present in omnidirectional images. In our previous work [23], we have suggested a new differential method adapted to omnidirectional images. This method is based on motion model designed for paracatadioptric images and an adapted neighborhood that takes into account the shape of the mirror. This method [23] uses a conventional gradient operator 
to compute image derivatives necessary for flow computation. This is why it was not appropriate for omnidirectional images.

- Optical flow computation using image processing on the sphere: The works of Daniilidis et al. [24], Tosic et al. [25], Mochizuki et al.[26] and Bagnato et al. [27] belong to this category. Daniilidis et al. defined a gaussian function and gradient operator on the sphere to solve the optical flow equation defined also in the spherical coordinates by assuming that it is locally constant. Later, Tosic and al [25] used multiresolution representation employing Spherical Laplacian Pyramid to filter spherical images. They proposed a new local motion estimation algorithm by computing correlation between two spherical images of the scene. Mochizuki et al.[26] proposed a formulation of Horn-Schunck method [7] on the spherical images. They computed optical flow on the sphere that yields monocular disparity on the antipodal point on the equator. They used this estimation to compute the navigation direction. A recent work by Bagnato et al. [27] also deals with the problem of computing optical flow using a formulation on the sphere. The authors have extended an existing and recent TV-L1 variational method [28] and have proposed a new formulation of this method as a graph-based framework on the sphere. They have defined spherical differential operators and proved that it is more accurate than planar differential operators applied to omnidirectional images to compute optical flow especially near the poles in spherical images.

Most authors in Omnidirectional vision use the sphere as an appropriate domain for omnidirectional images. The spherical image processing will take 
into account the specific geometry of omnidirectional cameras [6] [24] [29] [25]. Our formulation of optical flow estimation is given on the sphere and the omnidirectional images are mapped onto a unit sphere and treated in the spherical domain. Then we propose to adapt approach based on multichanel decomposition of optical flow equation [19]. This method proved its efficiency in the case of perspective images and it was indicated that it gives the best results for optical flow estimation [18] [19] [17]. For this reason, we propose to use a spherical wavelet and decompose the optical flow equation on the sphere to estimate the optical flow field. The reminder of the paper is organized as follows. In section 2, we shall present the optical flow constraint equation on the sphere and the stereographic projection necessary for all transformations between the catadioptric image and the unit sphere. Section 3 describes our approach to compute optical flow using spherical wavelet to constrain the optical flow equation on the sphere. In section 4, the experimental results are given and comparative measurements are discussed for synthetic and real images. We shall eventually give our conclusion in section 5 and provide guidelines for future works.

\section{Optical flow constraint equation on the sphere}

Geyer and Daniilidis have introduced unifying theory for all central catadioptric sensors [30]. They proved that central catadioptric projection is equivalent to a central projection to a virtual sphere followed by projection from the sphere to the retina. This second projection depends on the shape of the mirror. Fig.(1) shows the equivalence between any catadioptric projection and the two-step mapping via the sphere. 
The parameter $\xi$ defines the shape of the mirror. In our case, we consider

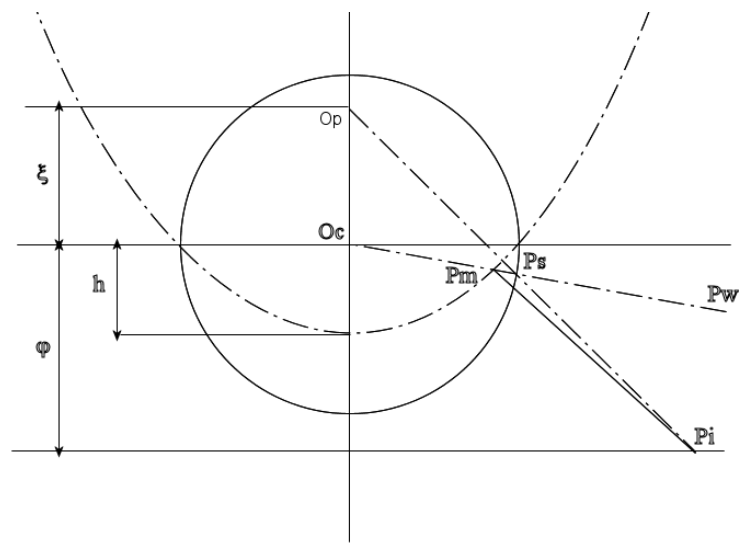

Figure 1: Equivalence between the catadioptric projection and the two-step mapping via the sphere.

parabolic mirror where $\xi=1$. However the method can easily be adapted to the general case. Let $P_{s}(\theta, \varphi)=P_{s}\left(X_{s}, Y_{s}, Z_{s}\right)$ be the point on the sphere, having a radius equal to one, where:

$$
\left\{\begin{array}{l}
X_{s}=\sin \theta \cos \varphi \\
Y_{s}=\sin \theta \sin \varphi \\
Z_{s}=\cos \theta
\end{array}\right.
$$

The stereographic projection of $P_{s}$ from the sphere to the catadioptric plane can be expressed on cartesian coordinates as:

$$
\left\{\begin{array}{l}
x=\frac{X_{s}}{1-Z_{s}} \\
y=\frac{Y_{s}}{1-Z_{s}}
\end{array}\right.
$$


Using eq.(2)and eq.(3), we obtain the image point $P i(x, y)$ expressed on spherical coordinates as:

$$
\left\{\begin{array}{l}
x=\cot \frac{\theta}{2} \cos \varphi \\
y=\cot \frac{\theta}{2} \sin \varphi
\end{array}\right.
$$

Note that the similar mapping model can easily be derived for all omnidirectional systems [30] [31]. In this paper, we look for a recovering of optical flow vectors on the sphere. For this reason, the omnidirectional images are mapped on the sphere and the formulation of Brightness Change Constraint Equation is rewritten on spherical coordinates. If $I_{s}(\theta, \varphi, t)$ denotes the temporal spherical image on the unit sphere $S^{2}$ and $\nabla I_{s}=\left(\frac{\partial I_{s}}{\partial \theta}, \frac{1}{\sin \theta} \frac{\partial I_{s}}{\partial \varphi}\right)^{T}$ denotes the spatial gradient vector on the sphere, the total derivative [24] gives:

$$
\frac{1}{\sin \theta} \frac{\partial I_{s}}{\partial \varphi} v_{\varphi}+\frac{\partial I_{s}}{\partial \theta} v_{\theta}+\frac{\partial I_{s}}{\partial t}=0
$$

This equation is equivalent to eq.(1) and represents the optical flow constraint equation on the unit sphere. The intensity of point on the sphere can be obtained by any interpolation of pixel intensities on catadioptric image. In our case, we used bicubic interpolation. $\left(v_{\theta}, v_{\varphi}\right)$ are the components of the flow vector in the tangential coordinates system. The partial derivatives of the spherical images can be obtained using the stereographic projection model (eq.(4)):

$$
\left[\begin{array}{c}
\frac{\partial I_{s}}{\partial \theta} \\
\frac{\partial I_{s}}{\partial \varphi}
\end{array}\right]=\left[\begin{array}{cc}
\frac{\partial x}{\partial \theta} & \frac{\partial y}{\partial \theta} \\
\frac{\partial x}{\partial \varphi} & \frac{\partial y}{\partial \varphi}
\end{array}\right]\left[\begin{array}{c}
\frac{\partial I}{\partial x} \\
\frac{\partial I}{\partial y}
\end{array}\right]=\left[\begin{array}{cc}
\frac{-\cos \varphi}{2 \sin ^{2} \frac{\theta}{2}} & \frac{-\sin \varphi}{2 \sin ^{2} \frac{\theta}{2}} \\
-\cot \frac{\theta}{2} \sin \varphi & \cot \frac{\theta}{2} \cos \varphi
\end{array}\right]\left[\begin{array}{c}
\frac{\partial I}{\partial x} \\
\frac{\partial I}{\partial y}
\end{array}\right]
$$

As in perspective case, we have a single equation to solve eq.(5) to determine two unknowns: $v_{\theta}$ and $v_{\varphi}$ ). An additional assumption must be given to solve 
the aperture problem on the sphere. Our proposed solution, detailed in the following section, consists in using a decomposition of spherical optical flow equation in spherical wavelets base assuming the constancy of optical flow over the support of the wavelets.

\section{Optical flow from multichannel spherical image decomposition}

We based our new approach on the work of Bruno et al.[19] and Bernard in [18]. It was proven in these references that multichannel decomposition of the optical flow equation gives robust estimation and a good compromise between robustness and time computation. Due to temporal aliasing, this method works well especially in multiscale framework [17] [18] [19]. This method consists in:

- Filtering an image sequence by a set of spatio-temporal filters or convolving an image sequence in wavelet base;

- Applying the optical flow constraint equation on each channel of the filter or of the wavelet.

The 2D Gabor filter used in [19] and the 2D discrete wavelets used in [18] are not appropriate to the distorted images given by catadioptric camera and should be adapted. Our proposed solution consists in using spherical wavelets base [29] to constrain the optical flow equation on the sphere. We chose the commonly used wavelet, Morlet wavelets [32], as a special case of Gabor wavelets. We first convolve the spherical optical flow equation with a set of spherical Morlet wavelets defined in different directions. The number of directions will then determine the size of the over-determined system to 
solve and get the optical flow vectors on the sphere. This convolution differs from the classical 2D convolution usually used for filtering images. In this section, we shall define a spherical wavelets and convolution on the sphere. Both definition will be used to constrain eq.(5).

\subsection{Spherical wavelets}

Our decomposition is based on directional spherical wavelets base. This choice of wavelets is motivated by the trust that wavelet bases are a welldesigned tool for our proposed approach. Wavelet bases have a natural multiscale structure. As a local frequency analysis tool, wavelets analysis favorably compares to filtering because it is far less computation intensive and still provides complete information on the signal. We based our spherical image decomposition on spherical wavelets proposed by Antoine et al. in [29]. They defined an interesting family of spherical wavelets which can be applied to any image represented in the spherical domain. Natural candidates in our purpose are directional wavelets on the sphere [32].

In [29] it was shown that any Euclidian wavelets can be mapped to sphere using an inverse stereographic projection. We chose the spherical Morlet wavelets $\psi_{M}$, represented in eq.(7) to decompose spherical images. $k 0$ is the norm of the wave vector of the wavelet in the Euclidian limit (accounting for the number of oscillations). $\varphi_{0}$ is the angle of the wavelet and $a$ is the scale of the wavelet. Note that this function is admissible for $k_{0}$ large enough $(>=6)$ [29]. Fig.(2) shows the spherical Morlet wavelet defined in two different directions on half-spheres.

$$
\psi_{M}(\theta, \varphi)=\frac{1}{2} \sqrt{\lambda} e^{i k_{0} \tan (\theta / 2) / \cos \left(\varphi-\varphi_{0}\right)} e^{-2 \tan (\theta / 2)^{2} / a^{2}}\left(1+\tan (\theta / 2)^{2} / a^{2}\right)
$$


with :

$$
\lambda=\frac{4 a^{2}}{\left(\left(a^{2}-1\right) \cos \theta+\left(a^{2}+1\right)\right)^{2}}
$$
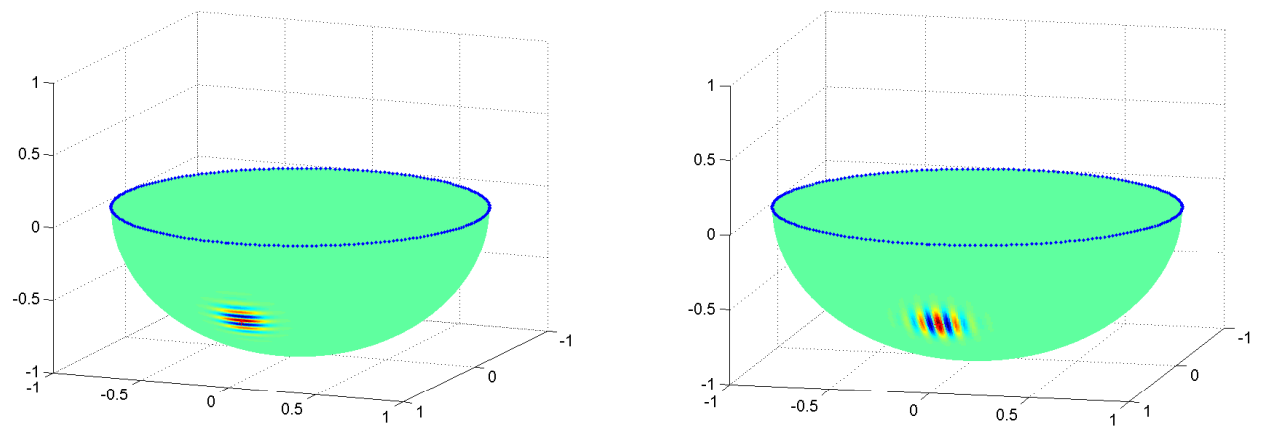

Figure 2: Spherical Morlet wavelet at scale $a=0.04$ and $\varphi_{0}=0$ (left) and $\varphi_{0}=\Pi / 2$ (Right)

\subsection{Wavelet Transform on the sphere}

Wavelet transformation is different from the Euclidean one. We shall present the definition of a convolution on the sphere. This convolution will be used to compute the spherical wavelet transform of images to calculate the optical flow vectors.

Let $\eta=(\theta, \varphi)$ be the point on the sphere $S^{2} . \Psi$ is the spherical wavelet defined on the sphere. If the rigid rotation $g$ is an element of the group of rotations $\mathrm{SO}(3)$ acting on the sphere $S^{2}, \Psi$ is rigidly transported on the sphere according to the operator $R_{g}$ as:

$$
R_{g} \Psi(\eta)=\Psi\left(g^{-1} \eta\right)
$$


The continuous wavelet transform of spherical image $I_{s}(\theta, \varphi)$ can be expressed as a convolution on the sphere with the spherical wavelet $\Psi$ as:

$$
W_{\Psi} I_{s}(\eta)=<I_{s}, R_{g} \Psi>
$$

The convolution on the sphere is defined by Driscoll and Healy [33] as:

$$
\left\langle I_{s}, R_{g} \Psi\right\rangle=\int_{S^{2}} I s(\eta) \overline{\Psi\left(g^{-1} \eta\right)} d \eta
$$

Where $: d \eta=\sin \theta d \theta d \varphi$. This convolution is performed in the Fourier domain using a simple multiplication like in $\mathbb{R}^{n}$. In practice, we will use the open source YAWTB Toolbox that contain an implementation of several directional wavelets on the sphere based on the work of Antoine et al. [29].

\subsection{Optical flow estimation}

In order to estimate the velocity vector at each point on the sphere using Eq.(5), we need to add some constraints to the spherical optical flow equation to solve the aperture problem. This assumption is based on the constancy of optical flow over the support of the spherical wavelets.

Let us consider the projection of the optical flow equation (eq.(5)) onto a wavelets base $\Psi_{i}(\theta, \varphi)$ (with $\mathrm{i}=1 \ldots \mathrm{N}$, and $\mathrm{N}$ is the number of wavelet directions). This allows to write :

$$
<\Psi_{M}(\theta, \varphi), \frac{1}{\sin \theta} \frac{\partial I_{s}}{\partial \varphi} v_{\varphi}+\frac{\partial I_{s}}{\partial \theta} v_{\theta}+\frac{\partial I_{s}}{\partial t}>=0
$$


Where $<>$ denotes the spherical convolution operator defined bellow in section(3.2). The multichannel decomposition approach is based on the assumption that $\left(v_{\theta}, v_{\varphi}\right)$ are constant over support of the wavelets $\Psi_{i}(\theta, \varphi)$ for all $i=1 \ldots N$. Note that the support of the wavelet must not be large to maintain the assumption of constant optical flow. Eq.(9) become:

$$
<\Psi_{M}(\theta, \varphi), \frac{1}{\sin \theta} \frac{\partial I_{s}}{\partial \varphi}>v_{\varphi}+<\Psi_{M}(\theta, \varphi), \frac{\partial I_{s}}{\partial \theta}>v_{\theta}+<\Psi_{M}(\theta, \varphi), \frac{\partial I_{s}}{\partial t}>=0
$$

Taking $\mathrm{N}$ directions for the directional wavelets, it leads a system of $2 * N$ equations since spherical Morlet wavelets are complex.

$$
\underbrace{\left(\begin{array}{c}
-r e a l<\Psi_{1}(\theta, \varphi), \frac{\partial I_{s}}{\partial t}> \\
-i m a g<\Psi_{1}(\theta, \varphi), \frac{\partial I_{s}}{\partial t}> \\
v_{\varphi}
\end{array}\right)}_{\left.\begin{array}{cc}
\text { real }<\Psi_{1}(\theta, \varphi), \frac{1}{\sin \theta} \frac{\partial I_{s}}{\partial \varphi}> & \text { real }<\Psi_{1}(\theta, \varphi), \frac{\partial I_{s}}{\partial \theta}> \\
\operatorname{imag}<\Psi_{1}(\theta, \varphi), \frac{1}{\sin \theta} \frac{\partial I_{s}}{\partial \varphi}> & i m a g<\Psi_{1}(\theta, \varphi), \frac{\partial I_{s}}{\partial \theta}> \\
\ldots . \\
\operatorname{real}<\Psi_{N}(\theta, \varphi), \frac{1}{\sin \theta} \frac{\partial I_{s}}{\partial \varphi}> & \operatorname{real}<\Psi_{N}(\theta, \varphi), \frac{\partial I_{s}}{\partial \theta}> \\
i m a g<\Psi_{N}(\theta, \varphi), \frac{1}{\sin \theta} \frac{\partial I_{s}}{\partial \varphi}> & i m a g<\Psi_{N}(\theta, \varphi), \frac{\partial I_{s}}{\partial \theta}>
\end{array}\right)}
$$

To solve this over-determined system for the two unknowns $v_{\theta}$ and $v_{\varphi}$, the least squares method is used. Therefore, the solution of (11) is given by 
minimizing:

$$
\begin{gathered}
\vec{V}=\arg \min _{v_{\theta}, v_{\varphi}} \sum_{i=1}^{N}\left[<\Psi_{i}(\theta, \varphi), \frac{1}{\sin \theta} \frac{\partial I_{s}}{\partial \varphi}>v_{\varphi}+<\Psi_{i}(\theta, \varphi), \frac{\partial I_{s}}{\partial \theta}>v_{\theta}\right. \\
\left.+<\Psi_{i}(\theta, \varphi), \frac{\partial I_{s}}{\partial t}>\right]^{2}
\end{gathered}
$$

If we denote the over-determined system by :

$$
\text { A. }\left[\begin{array}{l}
v_{\varphi} \\
v_{\theta}
\end{array}\right]=B
$$

The solution of the system is given as:

$$
\left[\begin{array}{l}
v_{\varphi} \\
v_{\theta}
\end{array}\right]=\left(A^{\prime} * A\right)^{-1} * A^{\prime} * B
$$

In practice, the linear system is solved with least square method. The estimated solution is taken into account according to two fixed thresholds. We compare the conditioning of a matrix $\left(A^{\prime} * A\right)$ with a first threshold. In addition, we test if the estimated solution $V=\left(v_{\varphi}, v_{\theta}\right)$ is acceptable or not by comparing the error $\|A V-B\|$ with a second fixed threshold. These two comparisons inform us on the stability of the linear system and allow to verify if our hypothesis of optical flow constancy is true or not and thus decide to take into account or not the estimated solution.

The least square estimation described above allows to determine a set of optical flow vectors defined on the tangential coordinate system $\left(v_{\theta}, v_{\varphi}, 0\right)$. To compare our optical flow field with that obtained using Bruno et al. method [19], this motion field must be projected on the catadioptric image using this 
transformation:

$$
\left[\begin{array}{l}
v_{x} \\
v_{y}
\end{array}\right]=\left[\begin{array}{cc}
\frac{\partial x}{\partial \theta} & \frac{\partial x}{\partial \varphi} \\
\frac{\partial y}{\partial \theta} & \frac{\partial y}{\partial \varphi}
\end{array}\right]\left[\begin{array}{c}
v_{\theta} \\
v_{\varphi}
\end{array}\right]
$$

Using eq.(4), the velocity vector $\left(v_{x}, v_{y}\right)$ in image space can be found by the following transformation of angular velocity $\left(v_{\theta}, v_{\varphi}\right)$ :

$$
\left[\begin{array}{l}
v_{x} \\
v_{y}
\end{array}\right]=\left[\begin{array}{cc}
\frac{-\cos \varphi}{2 \sin ^{2} \frac{\theta}{2}} & -\cot \frac{\theta}{2} \sin \varphi \\
\frac{-\sin \varphi}{2 \sin ^{2} \frac{\theta}{2}} & \cot \frac{\theta}{2} \cos \varphi
\end{array}\right]\left[\begin{array}{l}
v_{\theta} \\
v_{\varphi}
\end{array}\right]
$$

\section{Experiments and Results}

To show the improvement given by our proposed method, we will compare it to four previously published methods.

We will compare our method first to a similar method developed for perspective camera given by Bruno et al.[19]. This method is based on decomposition of optical flow equation using Gabor filter bank. The parameters of Gabor filter are fixed to preserve the stability of the linear system and to give the smallest error estimation. We have fixed experimentally the optimal parameters of Gabor filter according to the size of the movement applied to the virtual cameras. Figure (3) shows angular errors obtained for many values of scale $\sigma$, central frequency $f_{0}$ and the number of filters $N$. According to the motion in the synthetic sequences, the optimal Gabor filter banks parameters are sets as follows:

$$
\sigma \in[3.0,6.0], \quad f_{0}<0.14 \quad N=6 .
$$


In [19], the optical flow estimation is performed in multiresolution framework. We use image decomposition with Gabor filter bank defined in three level of multiresolution estimation where $G_{2^{-k} f_{0}, 2^{k} \sigma, N}$ corresponds to the Gabor filter bank in level $k$. Equivalently the spherical wavelets support is arranged to have the same size as that fixed in [19]. For this reason, we fixed a scale of wavelets in $a=0.05$ and $a=0.03$ respectively for large and small motion. For all sequences, we took six orientations of the spherical Morlet wavelets and 2D Gabor filter.

We have compared our proposed method also to that of Kanade et al. [14]. This method is still one of the most popular versions of two-frame differential methods for motion estimation and is often used in robotic applications. Lucas and Kanade compute the optical flow on a point $(x, y)$, considering that the motion is constant in a fixed neighborhood of this point.

The third method, which we will compare our proposed method with, is our previously published method dedicated to omnidirectional images [23]. This method is an adaptation of Lucas and Kanade's method which uses a new constrain taking into account the distortions existing in the omnidirectional images.

Finally, we will conduct comparison with the results obtained using a method developed by Danilidis et al. [24]. This method can be also considered as an adaptation of Lucas and Kanade's method. It uses the same assumption that the motion is constant in a fixed neighborhood. The main difference between this two adapted methods ([23] and [24]) is that the first one uses a new definition of neighborhood and new motion model to solve the aperture 

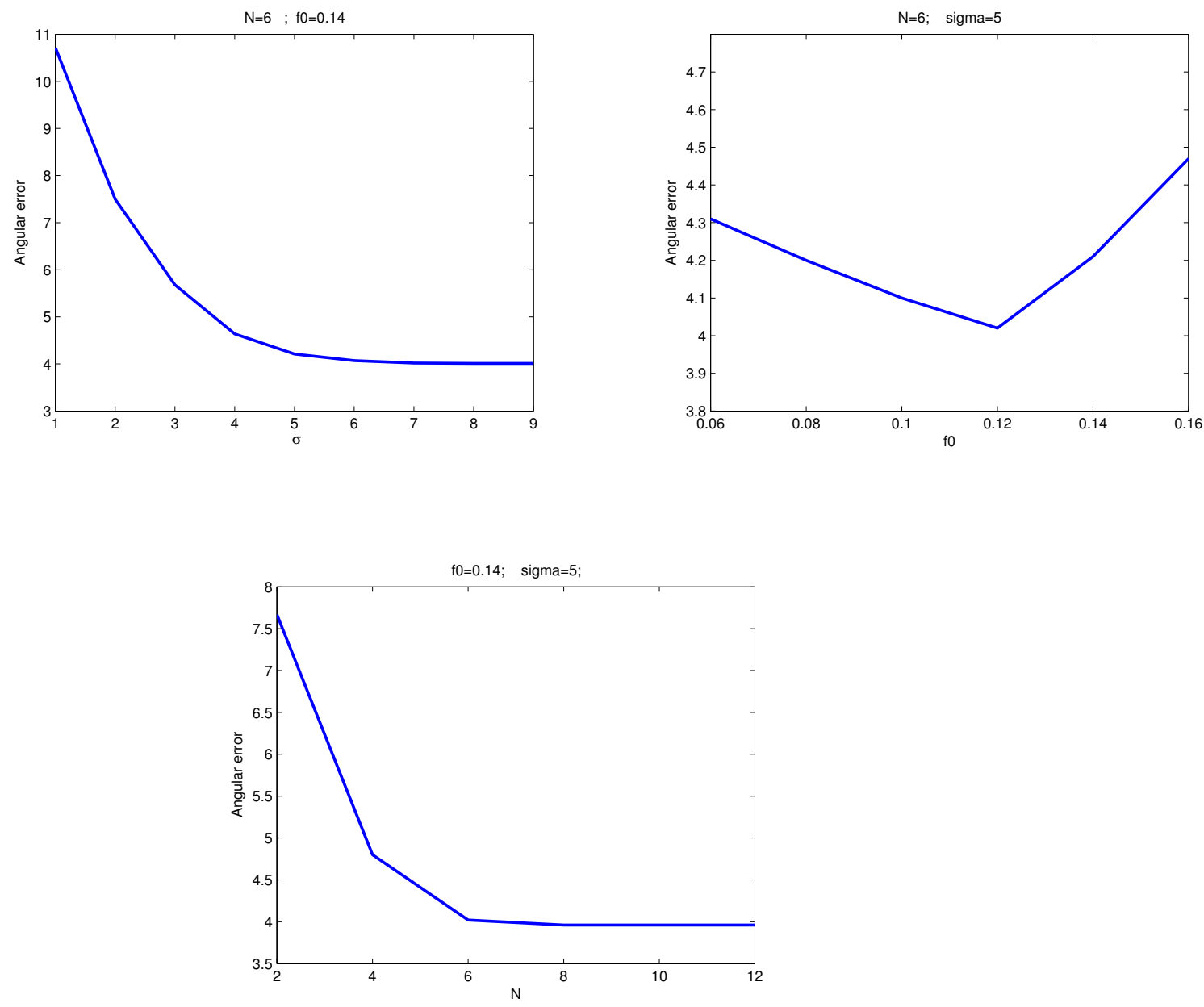

Figure 3: Top: Angular error in function of scale $\sigma\left(N=6 ; f_{0}=0.014\right)$. Middle: Angular error in function of central frequency $f_{0}(N=6 ; \sigma=5)$. Bottom: Angular error in function of number of filters $N\left(f_{0}=0.014 ; \sigma=5\right)$. These experiments are applied to the sequence obtained with translation $T=[-1 \mathrm{~cm}, 0,0]$ of virtual camera. 
problem on the image space, whereas the second one is based on the spherical representation of omnidirectional images and solves the same spherical optical flow equation that we use in this paper.

As presented in section 3.3, to test the stability of our linear estimation we fixed the conditioning threshold on 0.1 and the adequacy threshold of estimated solution in 0.1. This allows to do not take into account the aberrant estimation of optical flow. We have considered three kinds of motions: translation, rotation and combined motion to show the effectiveness of our contribution. These motions are applied to virtual and real cameras moving on a plane perpendicular to their optical axis.

\subsection{Synthetic sequences}

We have tested our algorithm on different synthetic sequences for which 2D motion fields are known. These sequences have been generated with POVRAY. A virtual parabolic camera observes four textured planes separated by approximatively $100 \mathrm{~cm}$. POVRAY generated images of $500 * 500$ pixels. An example of synthetic images is shown in (Fig.(4)). We have compared our proposed method to that proposed in [19] for perspective images and that proposed in [23] for catadioptric images. To assess the optical flow estimation methods, we use the mean of angular errors [20] between the known velocity vectors, $V_{r}=\left(u_{r}, v_{r}\right)$ and an estimated vectors $V_{e}=\left(u_{e}, v_{e}\right)$. This error measurement takes into account at the same time the error made on orientation and amplitude of the estimated vectors flow for each pixel of 
the image. It is given by:

$$
e=\arccos \left(\frac{u_{e} u_{r}+v_{e} v_{r}+1}{\sqrt{u_{r}^{2}+v_{r}^{2}+1} \sqrt{u_{e}^{2}+v_{e}^{2}+1}}\right)
$$

Fig.(5) shows the angular error map obtained for different camera motions.
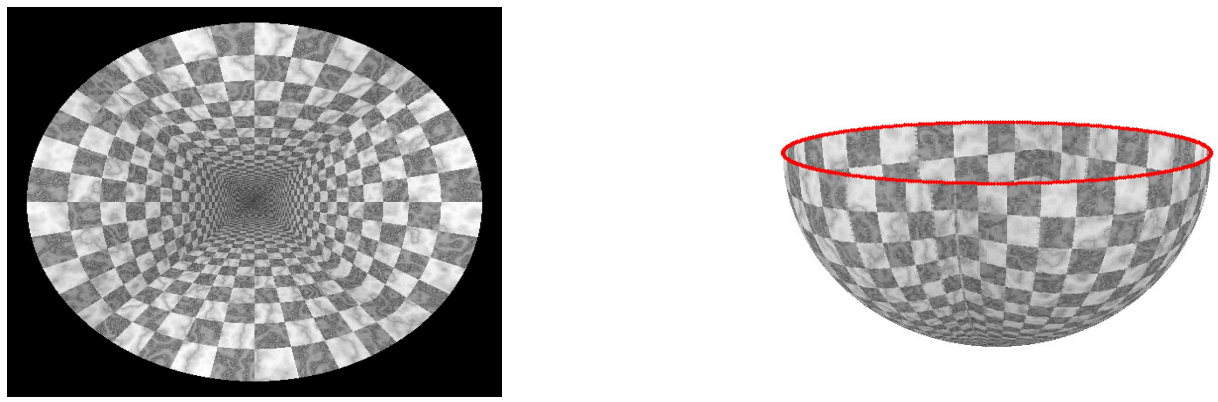

Figure 4: Left: Synthetic image composed by four textures plans and on right its spherical equivalent image

The blue color means an error of 0 when a green and red color reflects an important error. The optical flow fields are shown in Fig.(6), Fig.(7) and Fig.(8) using Bruno et al. and proposed methods. Note that in all these experimentations we do not consider the image points around the center of images. In these locations both methods generate significant errors. In the rest of the image space the flow vectors prove that the adapted approach on the sphere compute a succeeded velocity vectors even with large camera motion and also in different positions on the distorted omnidirectional images. This means that the fixed support of 2D Gabor filter is not appropriate to omnidirectional images. 
The mean angular errors obtained from different sequences using the five implemented methods are indicated in Tab.(1). These results show that the classical approaches ([19] and [14]), even if these methods work well for perspectives images, are not appropriate to omnidirectional images. Only the case of very small rotational motion of virtual camera gives an acceptable result using these methods.

The angular errors obtained using spherical methods ([24] and our proposed method in this paper) are inferior to that obtained using adapted method on the image space [23]. These results give the argument that methods dedicated to omnidirectional images are more robust when using a spherical model to represent images. Finally, we can see that angular errors are inferior using our spherical approach especially with large camera motions. This result proves that multichannel spherical images decomposition method proposed in this paper gives good estimation of local motion compared to differential methods [24] [23].

\subsection{Real sequences}

We have also used sequences of real omnidirectional images. The sequences are obtained using a camera which is mounted on a mobile robot and moves on a plane perpendicular to its optical axis. The camera is a SONY $D F W-S X 910$. We obtain an image of $1280 * 960$ pixels. The catadioptric sensor mounted in the mobile robot is illustrated in Fig.(9) with the image that it gives. The camera is calibrated using the Omnidirectional Calibration Toolbox [34]. Fig.(10) shows the optical flow fields for a rotation around Z-axis in real scene. Fig.(11) illustrates a combined motion of camera and shows the optical flow fields. Finally, Fig.(12) represents the optical flow 

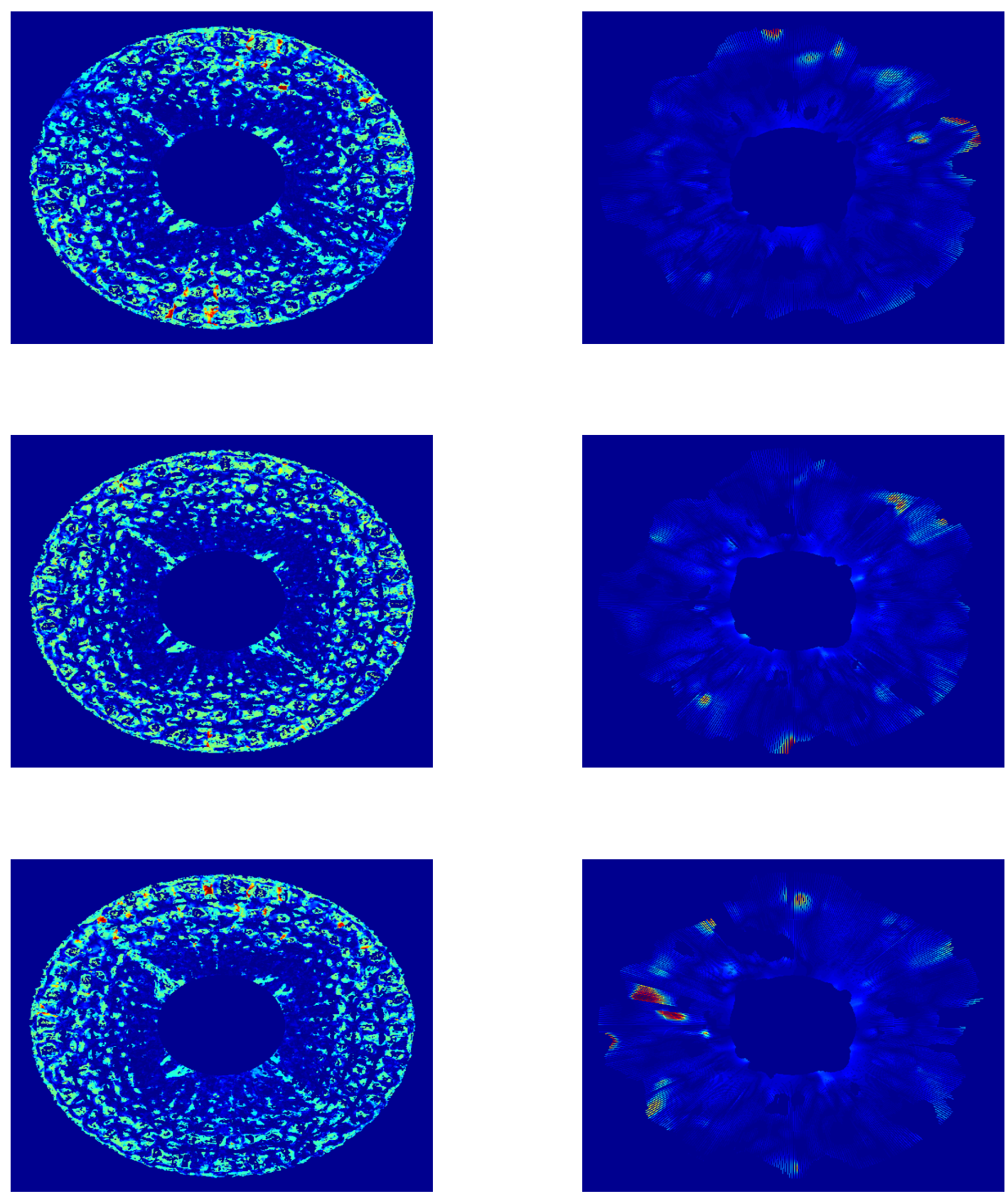

Figure 5: Angular Error Maps obtained using Bruno et al. method (left) and adapted method (right) for different kinds of motion: Top: Pure translation in $\mathrm{X}$ and Y-direction. Middle: Pure rotation of $1^{\circ}$ around Z-Axis. Bottom: combined motion 

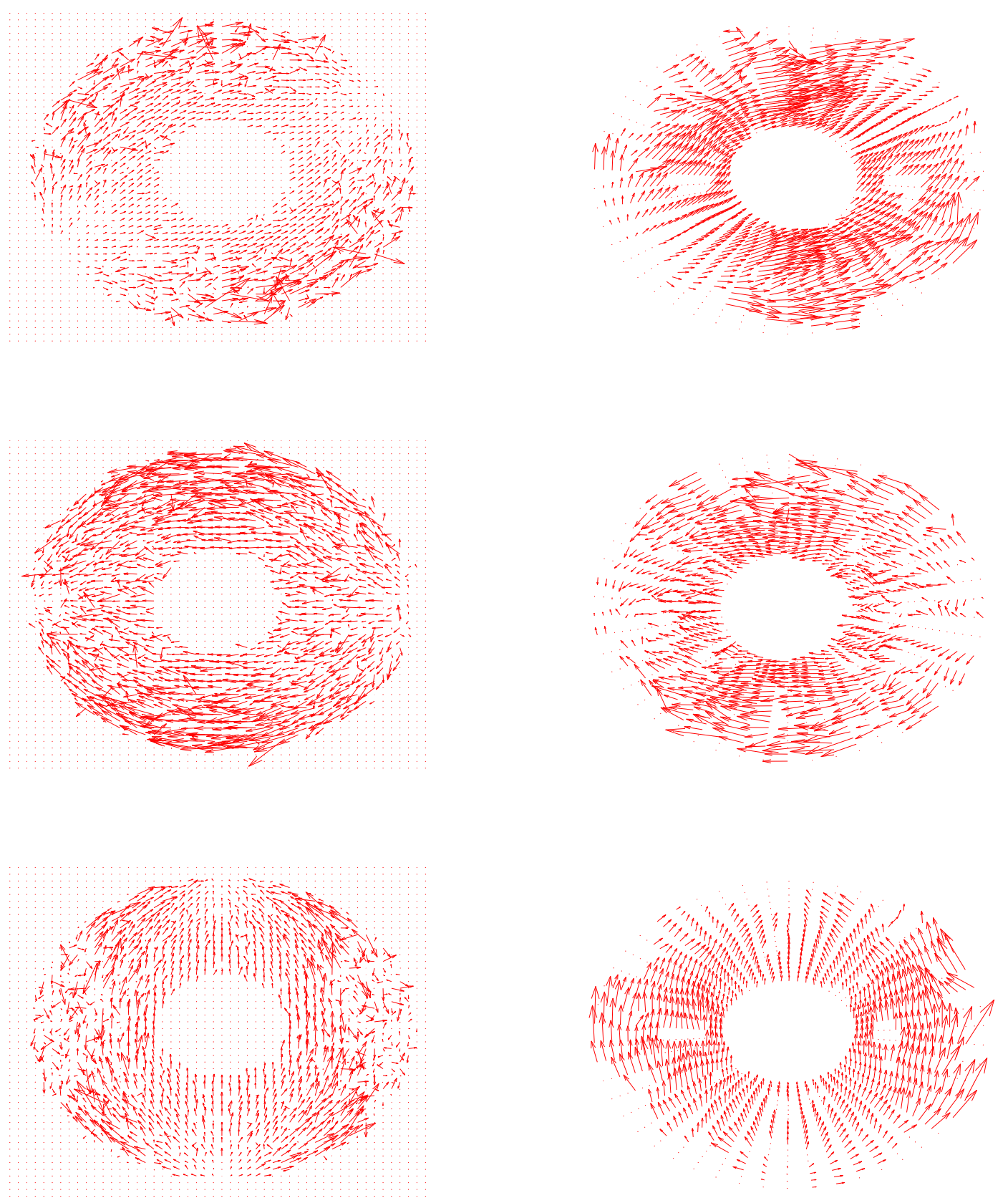

Figure 6: Optical flow fields from pure translation of camera. In the top: $T=$ $[5 \mathrm{~cm}, 3 \mathrm{~cm}, 0]$. In the middle: $T=[-1 \mathrm{~cm}, 0,0]$. In the bottom: $T=[0,3 \mathrm{~cm}, 0]$. The fields on the left correspond to the Bruno et al. method and those on the right to the adapted method. 

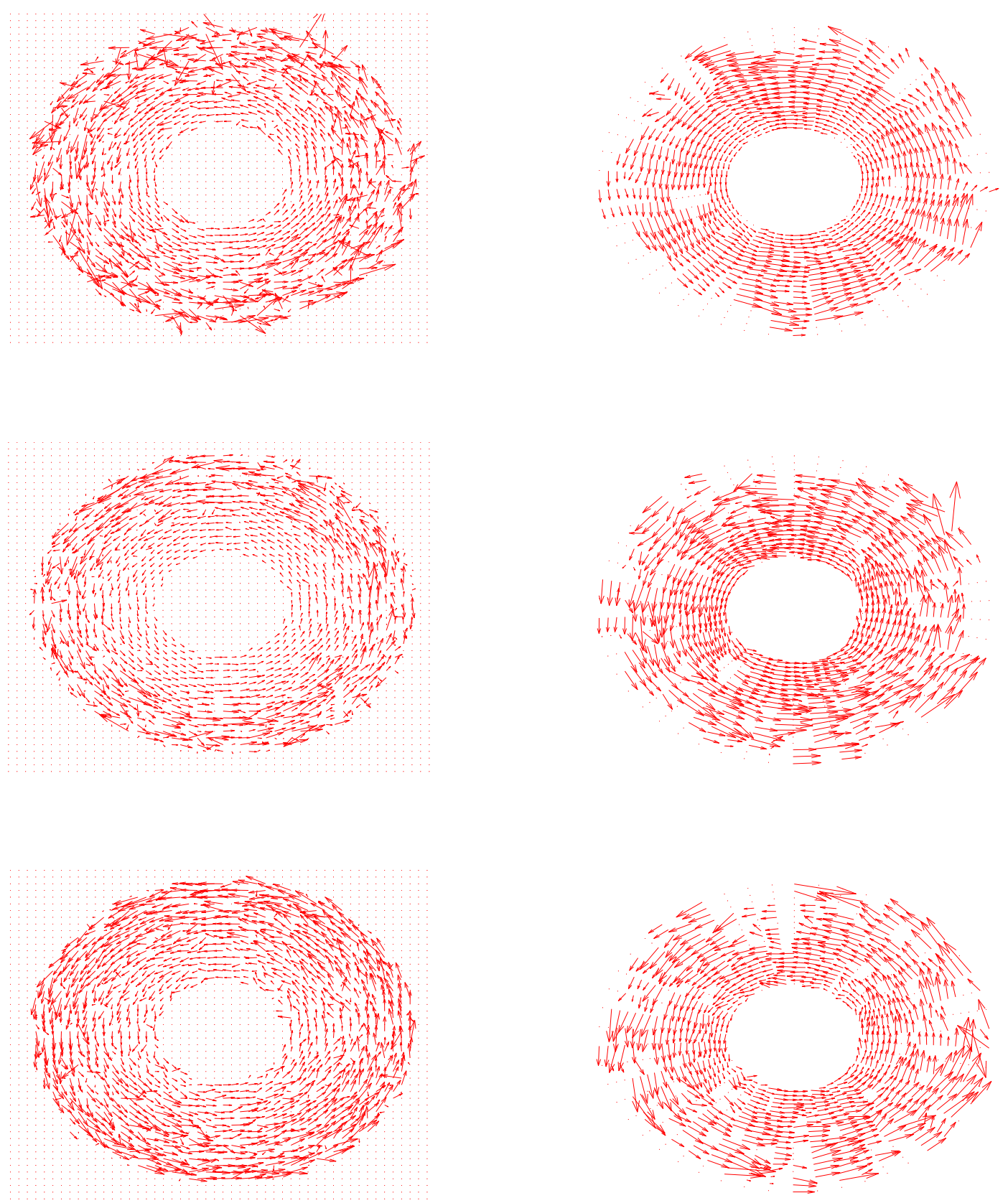

Figure 7: Optical flow fields from pure rotation of camera. At the top: $R=\left[0,0,2^{\circ}\right]$. In the middle: $R=\left[0,0,1^{\circ}\right]$. At the bottom: $R=\left[0,0,0.5^{\circ}\right]$. The fields on the left correspond to the Bruno et al. method and those on the right to the adapted method. 

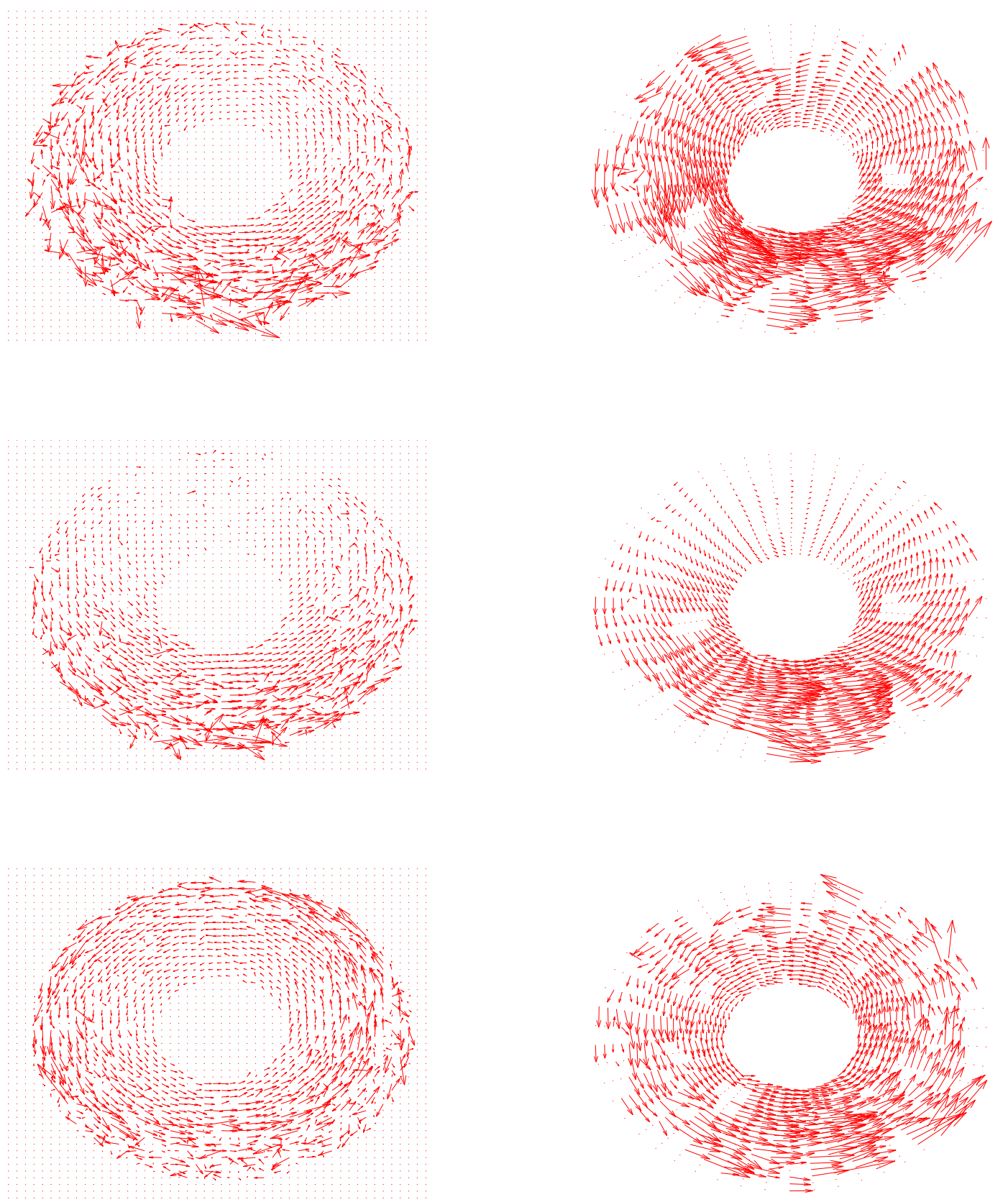

Figure 8: Optical flow fields from combined motion of the camera. At the top: $R=\left[0,0,2^{\circ}\right]$ and $T=[2 \mathrm{~cm},-1 \mathrm{~cm}, 0]$. In the middle: $R=\left[0,0,1^{\circ}\right]$ and $T=[2 \mathrm{~cm}, 0,0]$. At the bottom: $R=\left[0,0,1^{\circ}\right]$ and $T=[0.5 \mathrm{~cm}, 0.2 \mathrm{~cm}, 0]$. The fields on the left correspond to the Bruno et al. method and those on the right to the adapted method. 


\begin{tabular}{|c|c|c|c|c|c|c|}
\hline \multirow{2}{*}{\multicolumn{2}{|c|}{ Sequences }} & \multicolumn{2}{|c|}{$\begin{array}{l}\text { Classical } \\
\text { methods }\end{array}$} & \multirow{2}{*}{$\begin{array}{c}\text { Adapted } \\
\text { method on } \\
\text { the image space } \\
\text { Radgui } \\
\text { et al.[23] }\end{array}$} & \multicolumn{2}{|c|}{$\begin{array}{l}\text { Adapted } \\
\text { methods on } \\
\text { the sphere }\end{array}$} \\
\hline & & $\begin{array}{l}\text { Bruno } \\
\text { et al.[19] }\end{array}$ & $\begin{array}{l}\text { Kanade } \\
\text { et al.[14] }\end{array}$ & & $\begin{array}{l}\text { Danilidis } \\
\text { et al.[24] }\end{array}$ & $\begin{array}{c}\text { Our } \\
\text { approach }\end{array}$ \\
\hline \multirow{3}{*}{ 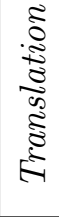 } & $T=[-1 \mathrm{~cm}, 0,0]$ & $8.28^{\circ}$ & $7.99^{\circ}$ & $5.81^{\circ}$ & $5.33^{\circ}$ & $4.94^{\circ}$ \\
\hline & $T=[0,3 \mathrm{~cm}, 0]$ & $16.00^{\circ}$ & $14.11^{\circ}$ & $8.63^{\circ}$ & $11.90^{\circ}$ & $4.56^{\circ}$ \\
\hline & $T=[5 \mathrm{~cm}, 3 \mathrm{~cm}, 0]$ & $14.62^{\circ}$ & $18.92^{\circ}$ & $16.46^{\circ}$ & $19.49^{\circ}$ & $4.71^{\circ}$ \\
\hline \multirow{3}{*}{ 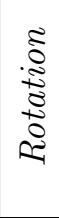 } & $R=\left[0,0,1^{\circ}\right]$ & $10.19^{\circ}$ & $8.60^{\circ}$ & $7.97^{\circ}$ & $7.59^{\circ}$ & $5.07^{\circ}$ \\
\hline & $R=\left[0,0,2^{\circ}\right]$ & $13.98^{\circ}$ & $16.73^{\circ}$ & $16.00^{\circ}$ & $12.91^{\circ}$ & $4.01^{\circ}$ \\
\hline & $R=\left[0,0,0.5^{\circ}\right]$ & $7.46^{\circ}$ & $5.61^{\circ}$ & $5.20^{\circ}$ & $7.50^{\circ}$ & $7.78^{\circ}$ \\
\hline \multirow{5}{*}{ 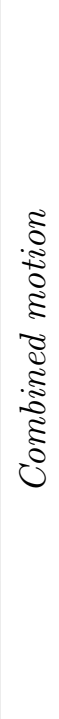 } & $\begin{array}{l}R=\left[0,0,0.5^{\circ}\right] \\
T=[0.3 \mathrm{~cm}, 0,0]\end{array}$ & $7.46^{\circ}$ & $8.97^{\circ}$ & $5.55^{\circ}$ & $5.73^{\circ}$ & $5.36^{\circ}$ \\
\hline & $\begin{array}{l}R=\left[0,0,1^{\circ}\right] \\
T=[2 c m, 0,0]\end{array}$ & $11.53^{\circ}$ & $15.39^{\circ}$ & $9.84^{\circ}$ & $7.71^{\circ}$ & $4.57^{\circ}$ \\
\hline & $\begin{array}{l}R=\left[0,0,1^{\circ}\right] \\
T=[0.5 \mathrm{~cm}, 0,0]\end{array}$ & $12.64^{\circ}$ & $11.33^{\circ}$ & $8.00^{\circ}$ & $7.40^{\circ}$ & $5.15^{\circ}$ \\
\hline & $\begin{array}{l}R=\left[0,0,1^{\circ}\right] \\
T=[0.5 \mathrm{~cm}, 0.2 \mathrm{~cm}, 0]\end{array}$ & $12.94^{\circ}$ & $11.35^{\circ}$ & $8.05^{\circ}$ & $7.28^{\circ}$ & $5.04^{\circ}$ \\
\hline & $\begin{array}{l}R=\left[0,0,2^{\circ}\right] \\
T=[2 \mathrm{~cm},-1 \mathrm{~cm}, 0]\end{array}$ & $14.14^{\circ}$ & $16.07^{\circ}$ & $15.70^{\circ}$ & $12.25^{\circ}$ & $5.70^{\circ}$ \\
\hline
\end{tabular}

Table 1: Mean of angular errors obtained for different synthetic sequences. 
fields obtained using a fixed omnidirectional camera and moving one object of the scene. In all these cases, the results show that our proposed method allows an estimation of accurate motion field in various scenes and different kinds of camera motions.

\section{Conclusion and future work}

In this paper we have described a new method to estimate optical flow vectors in omnidirectional images. This method uses the sphere as the space of image processing. We have used spherical wavelets to constrain the optical flow equation on the sphere. The simulation results in real and synthetic images shows that the proposed method allows an accurate estimation of optical flow for a wide range of camera motions.

As future works, we shall first try to estimate optical flow using multichannel decomposition in multiresolution frameworks. We trust that due to temporal aliasing, it will give more accurate optical flow vectors. On the other hand, we have observed that a number of other recent works in omnidirectional vision takes a traditional method like that of Lucas and Kanade [14] for optical flow estimation, even if it is not adapted, to recover the egomotion [6] [35] [36] or the epipoles [37] [38]. We believe that using our adapted optical flow field proposed in this paper, instead of the classical one, will improve the estimation of egomotion and epipoles in omnidirectional images.

\section{References}

[1] J. Kim, Y. Suga, An omnidirectional vision-based moving obstacle detection in mobile robot, International Journal of Control Automation and Systems 5 (6) (2007) 663-673. 

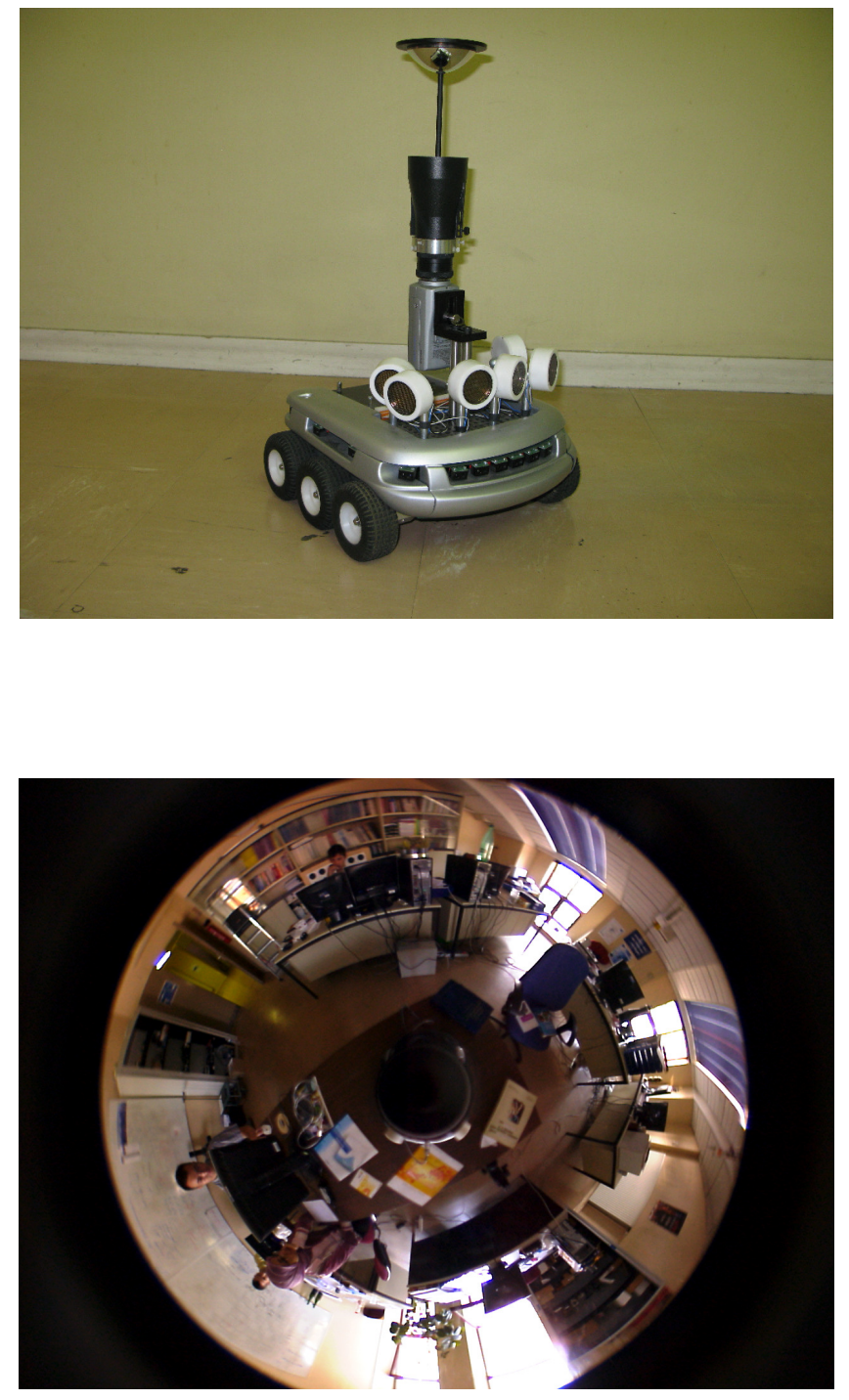

Figure 9: Omnidirectional camera mounted on mobile robot and the real image that it gives 

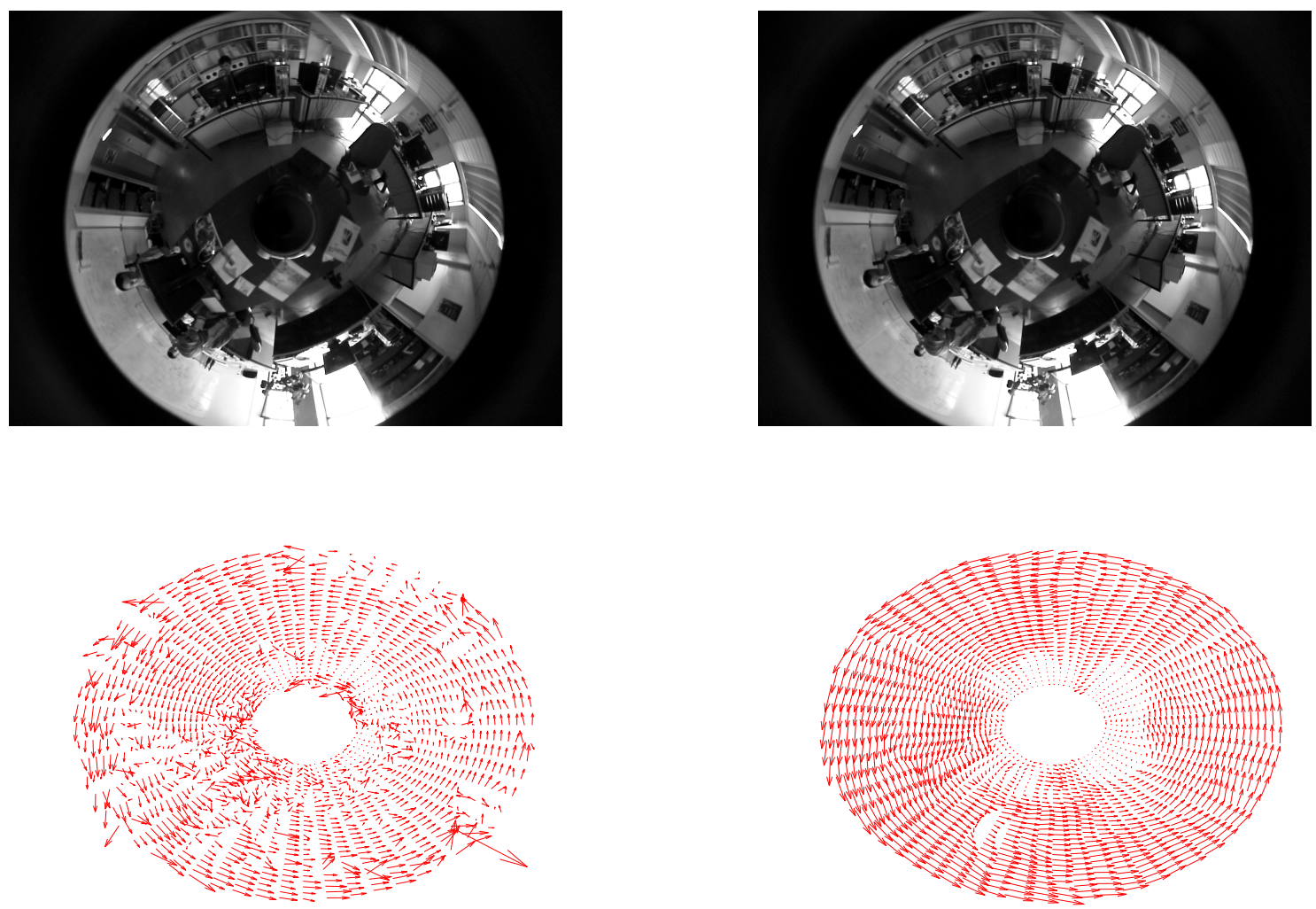

Figure 10: At the top : Two images from real sequence obtained using pure rotation around Z-axis. At the bottom: the flow fields using the Bruno et al. method on the left and the adapted method on the right. 

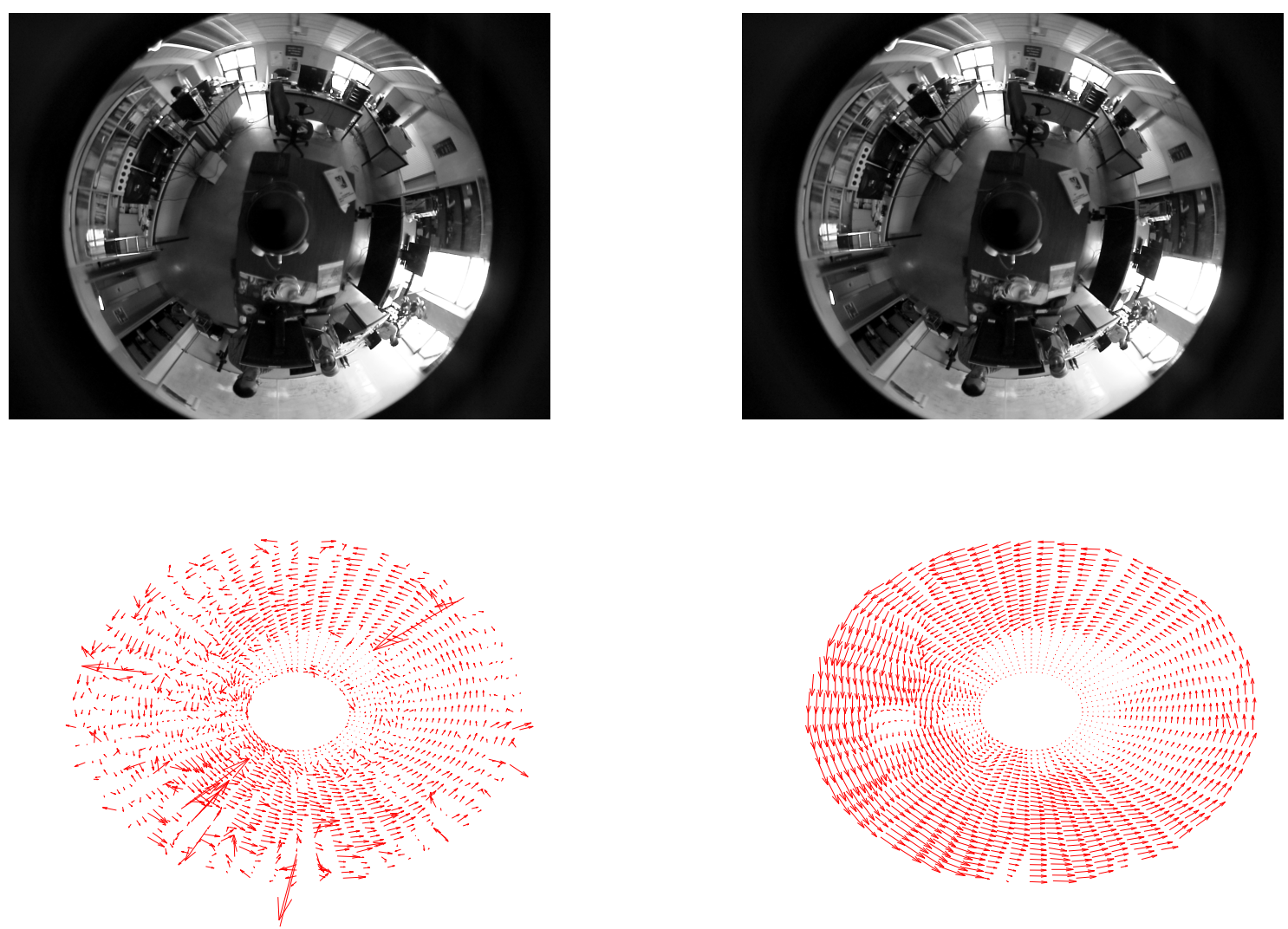

Figure 11: original images and optical flow fields obtained using combined motion of a translation along Y-axis and rotation around Z-axis from the Bruno et al. method (left) and the adapted method (right) 

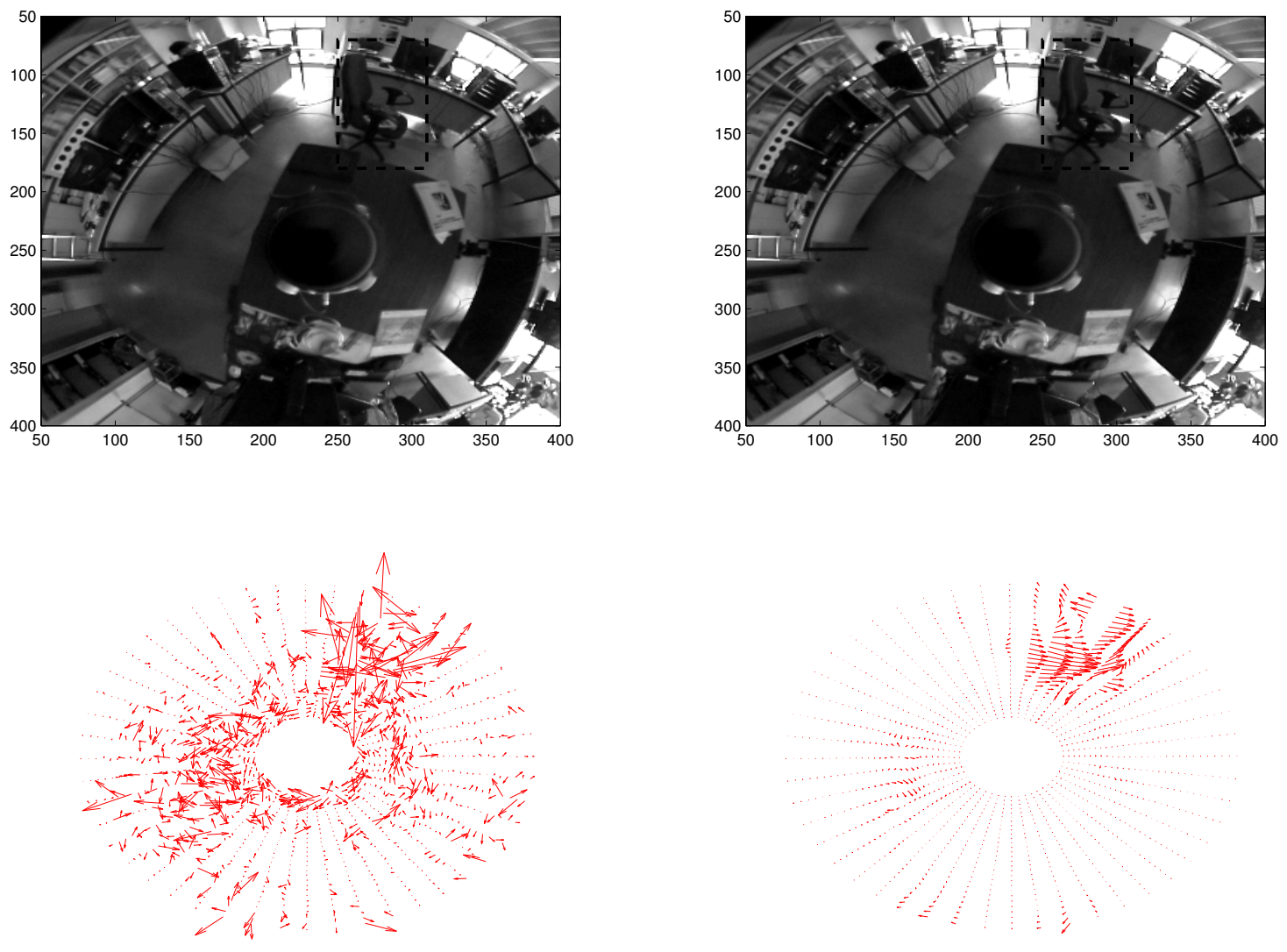

Figure 12: Optical flow fields obtained using a fixed camera and moving object in the scene. On the left, Bruno et al. method. On the right: adapted method 
[2] W. Yoshizaki, Y. Mochizuki, N. Ohnishi, A. Imiya, Free space detection from catadioptric omnidirectional images for visual navigation using optical flow, in: OMNIVIS08, 2008.

[3] M. L. Wang, C. C. Huang, H. Y. Lin, An intelligent surveillance system based on an omnidirectional vision sensor, in: IEEE Conference on Cybernetics and Intelligent Systems, 2006, pp. 1-6.

[4] R. Bunschoten, B. Krose, Visual odometry from an omnidirectional vision system, in: IEEE International Conference on Robotics and $\mathrm{Au}-$ tomation(ICRA'03), Vol. 1, 2003, pp. 577-583.

[5] N. Winters, J. Gaspar, G. Lacey, J. Santos-Victor, Omni-directional vision for robot navigation, in: Omnidirectional Vision, 2000. Proceedings. IEEE Workshop on, 2000, pp. 21-28.

[6] J. Gluckman, S. Nayar, Ego-motion and omnidirectional cameras, in: IEEE International Conference on Computer Vision (ICCV), 1998, pp. 999-1005.

[7] B. Horn, B. Schunck, Determining optical flow, Artificial Intelligence 17 (1-3) (1981) 185-203.

[8] P. Anandan, A computational framework and an algorithm for the measurement of visual motion 2 (1989) 283-310.

[9] E. Adelson, J. Bergen, Spatiotemporal energy models for the perception of motion, J. Opt. Soc. Am. A 2 (1985) 284-299. 
[10] D. Heeger, Optical flow using spatiotemporal filters, International Journal of Computer Vision 1 (4) (1988) 279-302.

[11] D. J. Fleet, A. D. Jepson, Computation of component image velocity from local phase information, International Journal of Computer Vision 5 (1) (1990) 77-104.

[12] C. Clifford, K. Langley, D. Fleet, Centre-frequency adaptive IIR temporal filters for phase-based image velocity estimation, Image Processing and its Applications 4-6 (1995) 173-177.

[13] T. Tsao, V. Chen, A neural scheme for optical flow computation based on gabor filters and generalized gradient method, Neurocomputing 6 (3) (1994) 305-325.

[14] T. Kanade, B. Lucas, An iterative image registration technique with an application to stereo vision, in: IJCAI81, 1981, pp. 674-679.

[15] H.-H. Nagel, On a constraint equation for the estimation of displacement rates in image sequences, IEEE Transaction on Pattern Analysis and Machine Intelligence 11 (1) (1989) 13-30.

[16] T. Burns, S. Rogers, D. Ruck, M. Oxley, Discrete, spatiotemporal, wavelet multiresolution analysis method for computing optical flow, Optical Engineering 33 (7) (1994) 2236-2247.

[17] J. Weber, J. Malik, Robust computation of optical-flow in a multiscale differential framework, International Journal of Computer Vision 14 (1) (1995) 67-81. 
[18] C. Bernard, Discrete wavelet analysis: A new framework for fast optic flow computation, in: ECCV98, 1998, p. II: 354.

[19] E. Bruno, D. Pellerin, Robust motion estimation using spatial gabor-like filters, Signal Processing 82 (2) (2002) 297-309.

[20] J. L. Barron, D. J. Fleet, S. S. Beauchemin, T. A. Burkitt, Performance of optical flow techniques, International Journal of Computer Vision 12 (1) (1994) 43-77.

[21] S. Baker, D. Scharstein, J. Lewis, A database and evaluation methodology for optical flow, in: IEEE international conference on computer vision (ICCV), 2007.

[22] C. Demonceaux, D. K. Akkouche, Optical flow estimation in omnidirectional images using wavelet approach, in: Conference of Computer Vision and Pattern Recognition Workshop, Vol. 7, 2003, p. page 76.

[23] A. Radgui, C. Demonceaux, M. Rziza, E. Mouaddib, D. Aboutajdine, An adapted lucas-kanade's method for optical flow estimation in catadioptric images, in: OMNIVIS08, 2008.

[24] K. Daniilidis, A. Makadia, T. Bulow, Image processing in catadioptric planes: Spatiotemporal derivatives and optical flow computation, in: Workshop on Omnidirectional Vision, 2002.

[25] I. Tosic, I. Bogdanova, P. Frossard, P. Vandergheynst, Multiresolution Motion Estimation for Omnidirectional Images, in: Proceedings of European Signal Processing Conference, 2005. 
[26] Y. Mochizuki, A. Imiya, Featureless visual navigation using optical flow of omnidirectional image sequence, in: Workshop of SIMPAR'08, 2008, pp. 307-318.

[27] L. Bagnato, P. Frossard, P. Vandergheynst, Optical flow and depth from motion for omnidirectional images using a tv-l1 variational framework on graphs, in: IEEE International Conference on Image Processing ICIP, 2009.

[28] C. Zach, T. Pock, H. Bischof, A duality based approach for realtime tv11 optical flow, in: DAGM07, 2007, pp. 214-223.

[29] J. Antoine, L. Demanet, L. Jacques, P. Vandergheynst, Wavelets on the sphere : Implementation and approximations, Applied and Computational Harmonic Analysis 13 (2002) 177-200.

[30] C. Geyer, K. Daniilidis, A unifying theory for central panoramic systems and practical implications, in: ECCV00, 2000, pp. II: 445-461.

[31] X. Ying, Z. Hu, Can we consider central catadioptric cameras and fisheye cameras within a unified imaging model?, in: European Conference on Computer Vision (ECCV04), Vol. 1, 2004, pp. 442-455.

[32] L. Demanet, P. Vandergheynst, Gabor wavelets on the sphere, in: SPIE Annual conference, 2003.

[33] J. R. Driscoll, D. M. Healy, Computing fourier transforms and convolutions on the 2-sphere, Adv. in Appl. Math. (1994) 202-250. 
[34] C. Mei, P. Rives, Single view point omnidirectional camera calibration from planar grids, in: IEEE International Conference on Robotics and Automation, 2007, pp. 3945-3950.

[35] J. Lim, N. Barnes, Directions of egomotion from antipodal points, in: IEEE Computer Society Conference on Computer Vision and Pattern Recognition, 2008.

[36] R. Vassallo, J. Santos Victor, H. Schneebeli, A general approach for egomotion estimation with omnidirectional images, in: OMNIVIS02, 2002, pp. 97-103.

[37] J. Lim, N. Barnes, Estimation of the epipole using optical flow at antipodal points, in: OMNIVIS07, 2007, pp. 1-6.

[38] J. Lim, N. Barnes, Estimation of the epipole using optical flow at antipodal points, Computer Vision and Image Understanding (2009). 